\title{
UNCERTAINTY OF THE ROLE OF CARBON CAPTURE AND SEQUESTRATION WITHIN CLIMATE CHANGE MITIGATION STRATEGIES.
}

\author{
Nico Bauer ${ }^{1, *}$, Ottmar Edenhofer ${ }^{1},{\text { Hermann } \text { Held }^{1} \& \text { Elmar Kriegler }}^{1}$ \\ ${ }^{1}$ Potsdam-Institute for Climate Impact Research, 14473 Potsdam, Telegraphenberg C4, Germany
}

\begin{abstract}
The optimal strategy and the implied costs of a stringent climate protection objective are uncertain and potentially large. The uncertainties arise from the growth and flexibility of energy demand and of low carbon energy supply. Carbon capture and sequestration is an option to reduce the economic costs of ambitious climate protection strategies, when energy demand and supply turns out to be inflexible with respect to the reduction of $\mathrm{CO}_{2}$ emissions. Unfortunately, the main characteristics of carbon capture and sequestration are uncertain, too. We use the integrated assessment model MIND to assess this issue. The model couples climate and economy. It computes the socially optimal extents and timings of the magnitude and structure of energy investments. In the base case $117 \mathrm{GtC}$ are captured and sequestered until 2050. Reduction of economic losses due to CCS are small relative to the overall economic losses. We assess the robustness of this result with respect to key parameters. For this purpose we use sensitivity and Monte Carlo analysis.
\end{abstract}

\section{Introduction}

There is some consensus that macro-economic mitigation costs increase substantially if ambitious climate protection goals should be achieved in order to avoid dangerous climate change. $\mathrm{CO}_{2}$ emissions from the combustion of fossil fuels is a major cause for climate change. In general, there are three technology routes to decrease $\mathrm{CO}_{2}$ emissions from the energy sector. First, to increase the efficiency of energy transformation and use. Second, to decrease the ratio of $\mathrm{CO}_{2}$ emissions per primary energy by shifting to carbon free energy technologies. Last, to capture $\mathrm{CO}_{2}$ at large scale industrial point sources and to store it in geological formations. We use the model MIND to assess these options and the associated uncertainties. In especially, we explore the economic significance of CCS within this portfolio of mitigation options.

In the first part we introduce the model MIND to the extent that is indispensable for this study. In the second part we investigate the optimal climate protection strategy and the rationale of balancing the mitigation options intertemporally. In the third part we analyse how robust these results really are. The paper ends with the conclusion.

\section{The Model MIND}

In the following we give a description of the model MIND, the Model of Investment and Technological Development. Model parameters that will be part of the uncertainty analysis are indicated with mathematical symbols. As far as numbers are explicitly mentioned in this section they are the default parameters of the base case. The base case serves as a reference that will be analysed in the next section. A more detailed description of the model and the justification of parameters can be found in [1].

The MIND model is a Ramsey-type growth model with endogenous technological development. It couples the economic system with the climate system by modelling the fossil energy sector and its carbon emissions explicitly. The energy demand can be reduced by either substituting it with capital and labour in a macro-economic production function or an endogenous increase of the energy productivity. The energy mix is determined by investments into the fossil and renewable energy sectors. The efficiency of investments into renewables increases through learning

*Corresponding author: Tel. (++49) 331-288 2535, Fax. (++49) 331-288 2642, Email: Nicolas.Bauer@pikpotsdam.de. We want to thank Claus Rachimow, Michael Flechsig and Uwe Böhm for the development and support of SimEnv and Michael Pahle for Matlab ${ }^{\circledR}$ support. Additionally, we want to thank Marian Leimbach and Kai Lessmann for inspiring discussions. 
by doing. Alternatively, the carbon emissions can be captured and stored in geological formations, which are subject to leakage.

The model determines optimal investment strategies into several capital stocks according to a social welfare function which depends on the stream of discounted utility per capita weighted with the population number. The population evolves exogenously and stabilises at $10.4 \mathrm{bil}$. in 2100 . Utility per capita depends on consumption per capita with diminishing returns. The investment paths are chosen as far as the resulting returns in terms of welfare improving consumption in the future are high enough. The task is to balance current and future interests via consumption-investment decisions.

Production of gross domestic product (GDP) requires the production factors labour, capital and energy. The three production factors are combined via a production function with a constant elasticity of substitution $\sigma=0.4$. The supply of labour is not determined endogenously within the model and equals the population. The main source of economic growth comes from investments in knowledge capital that improves the productivity of labour. The growth rate of labour productivity depends on the endogenous specific investment share devoted to this purpose The parameter $\alpha_{L}$ influences the overall productivity of the investment share. It is calibrated in order to reproduce a benchmark: when $2.5 \%$ of the GDP is devoted to these investments, then the labour productivity increases by $\alpha_{L} \%$. The productivity of energy is increased by an equivalent mechanism, but $\alpha_{E}$ is calibrated to the benchmark of a $1 \%$ GDP share. In both functions there are diminishing returns of productivity. The third production factor is capital, which is accumulated by investment and decreased by exponential depreciation.

Secondary energy supplied to the economy can be produced from fossil and renewable energy sources. The fossil energy sector demands capital and fossil primary energy carriers, which have to be extracted with capital. Moreover, there is an exogenous path of traditional non-carbon energy that comprises traditional renewables, large hydro and nuclear power. The extraction sector is subject to learning. The learning potential $\kappa_{r e s, l}^{\max }$ is limited to a doubling of the capital productivity relative to the present-day level. The learning effect competes with the scarcity effect that implies a decreasing capital productivity. When cumulative resource extraction reaches $\chi=3500 \mathrm{GtC}$, the capital productivity is a sixth of its initial value. The initial capital stocks in the fossil energy $K_{\text {fos }}^{0}$ and extraction sector $K_{\text {res }}^{0}$ are assumed to be 6 tril.\$US and 5 tril.\$US, respectively.

The renewable energy sector needs only capital. The investment costs into new capital vintages decrease according to a learning rate of $l r=15 \%$ for a doubling of installed capacity. The investment costs are divided in two parts. The first part are the fix floor costs assumed to be 500 \$US per $\mathrm{kW}$. The second part are the investment costs $c_{0}^{r e n}$ that can be reduced through learning by doing and are assumed to be $700 \$ \mathrm{US}$ per $\mathrm{kW}$.

There are $i=1, \ldots, 5$ different capture technologies: industrial hydrogen production, new coal, conventional coal, cement and iron\&steel. They are characterised by the constant investment cost $c_{i}^{\text {ccs }}$ in units of \$US per tC, the operation and maintenance O\&M costs in \$US per tC and year and the energy demand in terms of EJ per GtC. Each capture technology is constrained with respect to a maximum amount of capturable $\mathrm{CO}_{2}$. For each technology only a certain fraction $\phi_{i}^{\text {ccs }}$ of emissions of the BAU case are available for capture; the BAU case will be described below.

This approach reflects the idea that there are technologies with different characteristics; e.g. the low cost technology of capturing $\mathrm{CO}_{2}$ from industrial hydrogen production are only applicable to sources that account for less than $6 \%$ total $\mathrm{CO}_{2}$ emissions. All five $\mathrm{CCS}$ technologies have the potential to remove $41 \%$ of the $\mathrm{CO}_{2}$ emissions without climate policy.

Transportation and injection are assumed to require capital. The capital requirements are higher for off-shore sequestration sites. This is partly offset by the higher flow rate, since the operating conditions are better off-shore. The length of pipelines are determined by the free capacities of the sequestration alternatives.

There are $j=1, \ldots, 6$ different sequestration alternatives. There are off- and on-shore sequestration sites. Moreover we distinguish aquifers with and without traps as well as depleted gas fields. The model does not take into account the enhancing effect of $\mathrm{CO}_{2}$ for hydrocarbon production. The corresponding sequestration alternatives (coal seams 
and mature oil fields) are not integrated in the model. The cumulated amount of carbon that can be injected into the sites of a sequestration alternative is limited by its capacity $s_{C C S, j}^{\max }$. The accumulation of carbon in the sequestration alternatives has two effects. First, a constant fraction $\lambda_{C C S, j}$ of the remaining $\mathrm{CO}_{2}$ leaks into the atmosphere. Second, as the free capacities of each sequestration alternative decrease, the distances for pipeline transportation increase. The distances are determined between a minimum distance $\theta_{C C S}$ and a maximum distance. The actual value depends on the fraction of free relative to total capacity. The rationale is that closer sites will be used first. When the free capacity of a sequestration alternative is high, it is more likely to find a sequestration site close to a capture plant. The distances increase as the capacities are exhausted.

The energy sector and CCS are highly flexible with respect to the introduction of additional equipment. This implies that energy shortages will be offset by investments, when it is needed and the necessary investments provide sufficient returns. The CCS equipment is added to existing capital, but the investment rates per capture technology are limited in order to avoid exessively rapid introduction of the technology.

The climate model is taken from [4,6]. The energy balance model is tuned to reproduce the global mean temperature (GMT) over the next 100 years for several greenhouse gas (GHG) emission scenarios and a climate sensitivity of $2.8^{\circ} \mathrm{C}$. The benchmark is the MAGICC model that in turn is used to emulate complex coupled atmosphere-ocean general circulation models in IPCC TAR. The model takes care of the near-term cooling effect of sulphate aerosols, which are coupled to the $\mathrm{CO}_{2}$ emission from fossil energy carriers. The aerosols are removed, when the carbon is captured. The emissions of other GHG are fixed at their levels in 2000, which is optimistic relative to IPCC SRES scenarios.

The economic variables are evaluated at 5 years time steps within the time frame $1995-2300$. Although we are interested in a time horizon until 2100 , this long time horizon is chosen because the stock variables and their long term effects beyond 2100 have to be taken fully into account by the optimisation procedure.

\section{Result of the Base Case}

The base case comprises the results for the no-climate policy case called BAU-case and the case of an ambitious climate protection path called CPP-case, which constrains the future temperature increase. GMT is not allowed to increase more than $2^{\circ} \mathrm{C}$ above pre-industrial levels and faster than $0.2^{\circ} \mathrm{C}$ per decade. In this section we distinguish two different CPP cases: in the $\mathrm{CPP}^{+}$case CCS is an available option for climate protection, while it is no option in the alternative $\mathrm{CPP}^{\circ}$ case.

The BAU is characterised by high economic activity, which reaches a GDP of 442tril.\$US in 2100. This is equivalent to more than 14 times the level in 2000 . The primary energy demand rises up to $1.9 \mathrm{ZJ}$ in 2100 . These numbers are similar to the IPCC SRES-A1 scenario family; see [5]. At the end of the century the economy starts the transition towards a renewable energy system, since fossil energy carriers become scarce.

The left panel of Fig. 1 shows the secondary energy delivered to the remaining economy in the three cases. The upper three curves show the total energy supply. The total energy supply in the BAU case is a nearly linear growth path. In the CPP cases this growth path is interrupted around the middle of the century. The difference between the two CCP cases is very small. It is a bit higher in $\mathrm{CCP}^{+}$until 2035, but then $\mathrm{CPP}^{\circ}$ exhibits a higher energy production.

Fig. 1 about here, please.

The lower four lines show the secondary energy supply from renewable and fossil energy for the CPP cases. The amount lacking the sum of the corresponding total energy production is the exogenous path of traditional non-fossil energy. The $\mathrm{CPP}^{\circ}$ case relies much more on renewable energy sources, while in the $\mathrm{CCS}^{+}$case the economy uses more fossil energy sources. In $\mathrm{CPP}^{+}$the economy is able to defer the transition towards renewable technologies and to prolong the use of the relatively cheaper fossil fuels. The economy employs CCS as a temporary strategy in order to limit the economic burden of the transition to renewable technologies. This is the benefiting effect of CCS. In the $\mathrm{CPP}^{\circ}$ case the economy simply needs a greater amount of renewable energy. This induces the learning effects earlier 
and to a greater extent in order to reduce the costs. Therefore, in the $\mathrm{CPP}^{+}$case renewables will become competitive in a later stage. This is the crowding-out effect of CCS on renewables.

As indicated by the difference of fossil energy production between the $\mathrm{CPP}^{\circ}$ and the $\mathrm{CPP}^{+}$case, the time path of CCS is an inverted U. Capturing is introduced in 2010 for industrial hydrogen production. Starting in 2015 New Coal technologies are used for carbon capture. In 2040 the maximum at $4.3 \mathrm{GtC}$ per year is reached. CCS declines afterwards and becomes zero in 2100 . The cumulative amount of CCS until 2050 is $117 \mathrm{GtC}$. This time path requires the near to mid term availability of New Coal technologies as well as a large number of sufficiently leak-proof sequestration sites.

The right panel of Fig. 1 shows the GDP losses relative to the BAU case for the two CPP cases. The inverted U shape is due to the growing competitiveness of renewables because of the learning effect, but the particular shapes differ depending on the availability of CCS. The near and mid-term GDP losses are lower and reach a lower maximum in the $\mathrm{CPP}^{+}$case. The extent of employing the CCS option can be seen from that picture: the less CCS allows to defer GDP losses and the faster learning takes place, the less carbon will be captured and the less fossil energy carriers will be used. Therefore, the problem is to balance the two competing effects. This is the optimal intertemporal allocation of climate protection options.

The relevance of the intertemporal allocation and its effect on the time path of CCS is best seen by contrasting the time paths in the right panel of Fig. 1 with the cumulative numbers. Discounting the GDP paths with 5\% results in $0.54 \%$ GDP losses relative to BAU for $\mathrm{CPP}^{\circ}$ and $0.53 \%$ for $\mathrm{CPP}^{+}$over the $21^{\text {st }}$ century. Therefore, the relevance of $\mathrm{CCS}$ is to defer rather than to reduce the GDP losses of a climate stabilisation policy.

The reduction of GDP losses due to CCS is relatively small. The maximum relative GDP loss in the $\mathrm{CPP}^{\circ}$ case is $1.56 \%$ in 2040 . The maximum reduction of GDP loss by employing CCS is $0.18 \%$ in 2035 . This gain should be contrasted with the $117 \mathrm{GtC}$ that have to be sequestered until 2050. In the next section we study the robustness of this result with respect to the uncertainty of parameter values.

\section{Results of the Uncertainty Analysis}

The intertemporal allocation problem suggests that the amount of CCS might be extremely sensitive with respect to parameter changes. Small parameter changes affect the relative weight of the benefiting and the crowding-out effect, which might lead to large changes of the amount of CCS, but to only small changes in GDP losses. In such a setting, sensitivity and Monte Carlo analysis are of interest. We use sensitivity analysis to find out whether and at what magnitudes uncertain, exogenous parameters tip the scales of the amount of carbon to be captured and sequestered. Moreover, the the consequences on the GDP losses evaluated. Sensitivity analysis is practical, when up to two parameters are varied at a time. We employ Monte-Carlo analysis to vary multiple parameters at a time. We note that the parameter changes affect the BAU case, too.

In the following we focus on the $\mathrm{BAU}$ and $\mathrm{CPP}^{+}$cases, only. We investigate the sensitivity with respect to two indicators: for the period 2000 - 2050 we compute the cumulative CCS in GtC and 5\% p.a. discounted cumulative GDP losses relative to the BAU case. For the sake of brevity we call the former CCS amount and the later GDP loss in the following.

Sensitivity and Monte Carlo analysis need a great number of single runs of a model. MIND is relatively cheap in terms of computational requirements. We embedded the model into the multi-run simulation environment SimEnv developed at PIK that enables the researcher to undertake sensitivity and Monte Carlo analysis on a massive parallel computing machine $[2,3]$.

\section{Sensitivity Analysis}

Sensitivity analysis is the systematic exploration of a grid of a subspace of a limited number of parameters. We focus on two sensitivity analysis with two key parameter variations for each. We first motivate the selection of the parameters and present the results. 
In the first sensitivity analysis we focus on key characteristics of CCS and the renewable technologies, because these two technologies are in competition for the supply of low carbon energy. The key characteristic of the renewable energy technologies is the learning process which reduces the investment costs. The key characteristic of the CCS technology is ability of sequestration sites to keep injected $\mathrm{CO}_{2}$ underground. This determines the environmental effectiveness of the technology. Therefore, in the first sensitivity analysis we focus on variations of the learning rate of renewables $l r$ and the leakage rate $\lambda_{C C S}$. For $\lambda_{C C S}$ we assume identical values for all sequestration alternatives.

Fig. 2 shows the sensitivity surface for CCS in the left and for GDP losses in the right panel for varying $l r$ and $\lambda_{C C S}$ over a considerable range and at a fine grid. For $l r$ the variation is from $0-35 \%$ with $1 \%$ steps. For $\lambda_{C C S}$ the variation is from $0-1 \%$ at $0.025 \%$ steps. This covers the overall range of parameter values that seem plausible. Each grid point in the surface represents a single run. The intensity of gray corresponds with the z-axis.

Fig. 2 about here, please.

We want to stress that the surfaces for both indicators are quite smooth. This indicates that the model is numerically reliable for this sensitivity. In Fig. 3 we observe that the amount of CCS varies over a considerable range, when varying $l r$ and $\lambda_{C C S}$. The sensitivity is monotonous over the entire grid. There are relatively flat regions, where parameter variations in either direction imply small changes of the CCS activity. A broad range of parameter variations is compatible with $85-125 \mathrm{GtC}$ of CCS amount.

There are critical regions, where CCS strongly changes with small parameter variations. The most pronounced one is found for moving towards low leakage and learning rates, which raises CCS up to $\sim 200 \mathrm{GtC}$. There are critical parameter combinations that delimit two regions with very different CCS amounts. For cases that belong to this plateau the amount of CCS is above $7 \mathrm{GtC}$ p.a. in 2050. It needs further research to clarify the reasons that lead to such non-linear model behaviour.

The right panel in Fig. 2 shows that the GDP losses behave quite differently. The step wise behaviour is not present any more and the leakage rate is not as important as the learning rate is. The leakage rate has an influence on the GDP losses, when the learning and the leakage rates are small. This region corresponds with the $200 \mathrm{GtC}$ plateau. Obviously, the learning rate is the more important parameter. Moreover, increasing the learning rate by $1 \%$ point leads to relatively greater benefits considering a low learning rate. This indicates diminishing returns effect of the GDP losses with respect to the learning rate.

The second sensitivity analysis is related to right panel of Fig. 1, where the shape of the relative welfare losses are in close relation with the difference of the energy supply between the CPP cases and the BAU case. The larger the difference of the energy supply relative to the BAU case the larger is the relative GDP loss. This property of the optimal climate protection strategy raises the question whether the economic impacts are mainly determined through the dynamics and flexibility of energy demand. Therefore, the second sensitivity analysis addresses parameters $\alpha_{L}$ and $\alpha_{E}$, which represent the productivity of endogenous improvements of labour and energy productivity.

Fig. 3 shows the sensitivity analysis for variations of the parameters $\alpha_{L}$ and $\alpha_{E}$. For $\alpha_{L}$ the variations are from 1 to $2.5 \%$ at $0.05 \%$ steps and for $\alpha_{E}$ from 1.2 to $2.5 \%$ at $0.05 \%$. The ranges of both parameters cover the variety of the IPCC SRES scenarios with respect to energy consumption and global GDP.

The surfaces look quite smooth and are monotonous. Both indicators react approximately linear to variations in both parameters. When $\alpha_{L}$ increases, the economy grows faster. This leads to higher energy demand and therefore the need for CCS increases. A counteracting effect is that a more affluent economy has a higher ability to bear the renewable energy investments to realise the learning effects. Obviously, the former effect outweighs the latter. When $\alpha_{E}$ decreases, the energy demand and therefore the amount of CCS increases. The overall impression getting from these surfaces is that the amount of CCS is more sensitive to $\alpha_{L}$, while the GDP losses depend nearly equally on both parameters. 
The sensitivity of both indicators to $\alpha_{L}$ and $\alpha_{E}$ is not as great as for $l r$ and $\lambda_{C C S}$. This result indicates that efforts for the improvement of knowledge should focus more on specific details of energy supply technologies rather than on economy wide influences. The next subsection qualifies this conclusion.

Fig. 3 about here, please

\section{Monte Carlo Analysis}

The base case and the sensitivity analysis of the CCS amount for both parameter variations deliver information for a Monte Carlo analysis (MCA). The base case suggests $117 \mathrm{GtC}$ should be captured and sequestered until 2050. The sensitivity analysis suggests that this result is robust. The sensitivity surfaces suggest that the spreading of frequencies of a MCA would be moderate around the base case. The first question is whether the result is still robust when a large number of parameters are varied. The second question is whether there are additional plateaus of cumulative amounts of CCS around the base case parameter set, where frequencies tend to accumulate. This would induce bimodal distributions. In Fig. 2 such behaviour is reasonable for the high plateau of $200 \mathrm{GtC}$, when the parameters have sufficient variance and are positively correlated.

Tab. 1: about here.

With the following 18-dimensional MCA we explore a space of uncertain parameters. For each parameter we have specified a normal distribution of possible values based on ad-hoc assumptions. To test the sensitivity of our results to the choice of input distribution functions, we also studied the case of log-normal input distributions with the same mean and variances and with doubled variance. For the following ensembles we draw samples of 5000 realisations. For this task again we use SimEnv, which offers the ability to perform Latin-Hypercube sampling.

In the left panel of Fig. 4 the frequency distribution for the normal distributions of parameters is given. As expected the mode of the distribution function is centered at $115 \mathrm{GtC}$. The mean and the median are within the same bin. The standard deviation is $36.4 \mathrm{GtC}$. The frequency distribution for the case of a log-normal distribution with the same variance is similar and therefore not shown. The most frequent bin is located at $105 \mathrm{GtC}$ and the standard deviation is a bit lower $(35.5 \mathrm{GtC})$. The right panel of Fig. 4 shows the frequency distribution the log-normal distribution with doubled variances. The frequency is distributed more unevenly, which is summarised in the standard deviation of $57.3 \mathrm{GtC}$.

When the model is linear, the doubling of the input parameter variances of a MCA would imply a doubling of the output variance. In our case however, the variance of the emerging distribution function increases by a larger factor than the doubling in the input parameter variances. This implies that the model represents a non-linear mapping of the model parameters on model variables.

The shape of the frequency distributions gives an interesting explanation for the increase of the variance. Of all cases $10 \%$ have CCS below $30 \mathrm{GtC}$, while Fig. 2 suggests the accumulation - if any - of frequencies at $200 \mathrm{GtC}$. Up to now, it is unclear whether there is a single parameter leading to this result or whether a cluster is the reason. In the case of a single parameter this would imply that a particular bottleneck should be obeyed. If a parameter cluster can be identified, it is reasonable to find out whether such a setting is reasonable or not.

Fig 4: about here, please.

These results imply a more integrated uncertainty analysis, which goes beyond the narrow insights obtained from the sensitivity analysis in Fig. 2. In fact, the resulting uncertainty of the optimal amount of CCS until 2050 obtained from the 18-dimensional MCA is found considerably larger than for the 2-D sensitivity study. A way to reduce the uncertainty is to remove input parameter sets and endogenous paths from the ensemble that are not reasonable.

\section{Conclusion}

The climate protection strategy comprises several overlapping phases. In each phase the three options for climate protection are employed at different intensities. In the near-term phase, the increasing energy productivity reduces 
emissions. Renewable energy technologies are introduced at a small scale in order to realise the learning effect. When renewables become more competitive the transition becomes sustainable in the long run. CCS is a near to mid-term option that allows more efficient investments related to fossil energy carriers with reduced emissions. The macro-economic mitigation costs can be reduced by CCS in the near to mid term. The cumulated economic effect over the $21^{\text {st }}$ century is small relative to the overall economic loss of the climate protection goal.

Due to the sensitivity analysis we have identified regions, where parameter variations in either direction imply small changes of the CCS activity. A broad range of variations of these two parameters is compatible with 85-125 GtC of CCS until 2050. There are critical regions where CCS strongly changes with small parameter variations. The Monte Carlo analysis puts the robustness of this result into perspective. The uncertainty appears to be larger, when all 18 uncertain parameters are varied. This indicates that an investment strategy robust under uncertainty is needed.

Our results have important consequences for the design of a regulatory framework for CCS (Edenhofer, Held, Bauer 2004). The amount of CCS is to inter alia determined by our innovative abilities that are uncertain. Over the next decade we will learn more about the corresponding parameters gained by large scale experiments. At the very beginning of these experiments a regulatory framework is needed that induces incentives for private firms, investors and engineers to look for sequestration sites with the very low leakage rates.

\section{Acknowledgements}

The financial support from the Volkswagen-Foundation is thankfully acknowleged.

\section{References}

1. Edenhofer, O., N. Bauer, and E. Kriegler, Climate Protection and Welfare: Insights from the Model MIND. Ecological Economics, submitted.

2. Edenhofer, O., N. Bauer, and H. Held, A Regulatory Framework for Carbon Capturing and Sequestration within the post-Kyoto Process, submitted to GHGT-7 conference peer review.

2. $\quad$ Flechsig, M., et al., The Multi-Run Simulation Environment SimEnv. User's Guide.. 2004, Potsdam Institute for Climate Impact Research.

3. Flechsig, M., et al. Techniques for Quality Assurance of Models in a Multi-Run Simulation Environment. in 4th International Conference on Sensitivity Analysis of Model Output. 2004. Santa Fe, U.S.A.

4. Kriegler, E. and T. Bruckner, Sensitivity of Emissions Corridors for the 21st Century. Climatic Change, 2004. in press.

5. Nakicenovic, N. and R. Swart, eds. Emissions Scenarios. Special Report of the Intergovernmental Panel on Climate Change. Special Reports, ed. IPCC. 2000, Cambridge University Press, UK: Cambridge, UK. 570.

6. $\quad$ Petschel-Held, G., et al., The Tolerable Windows Approach: Theoretical and Methodological Foundation. Climatic Change, 1999. 41: p. 303-331.
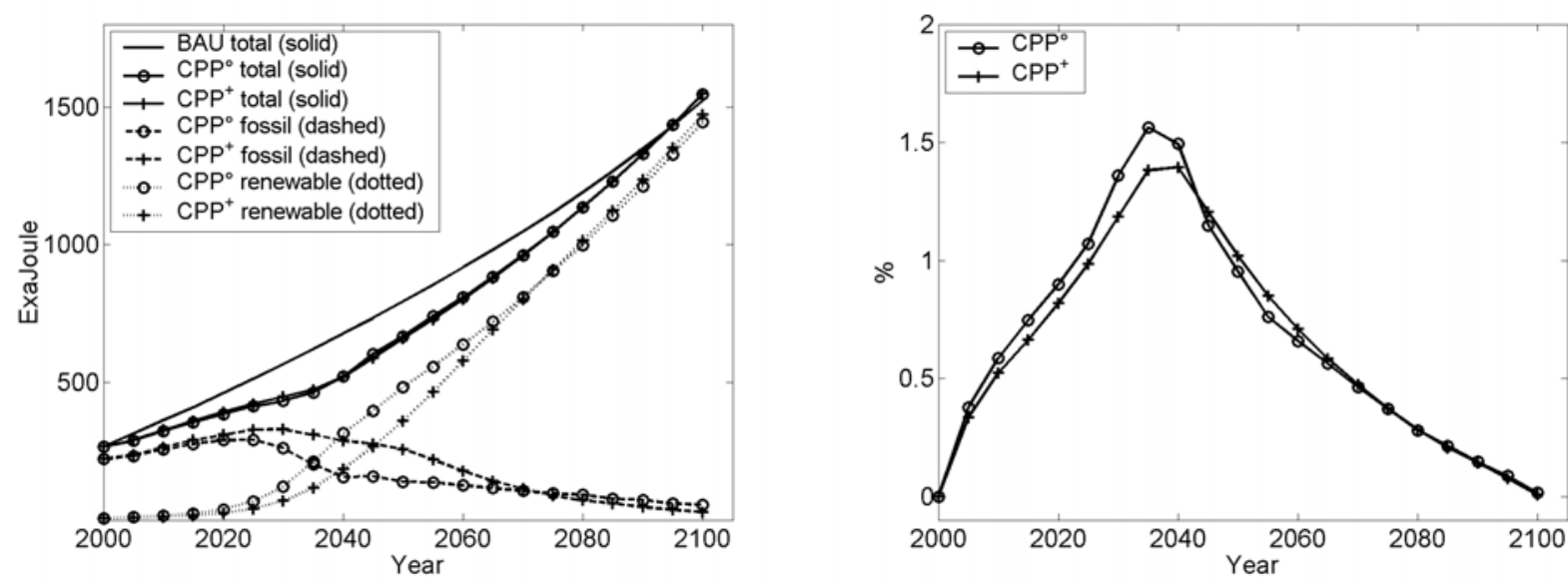
Fig. 1: Secondary energy production (left panel). GDP losses relative to BAU $2000-2100$ (right panel).
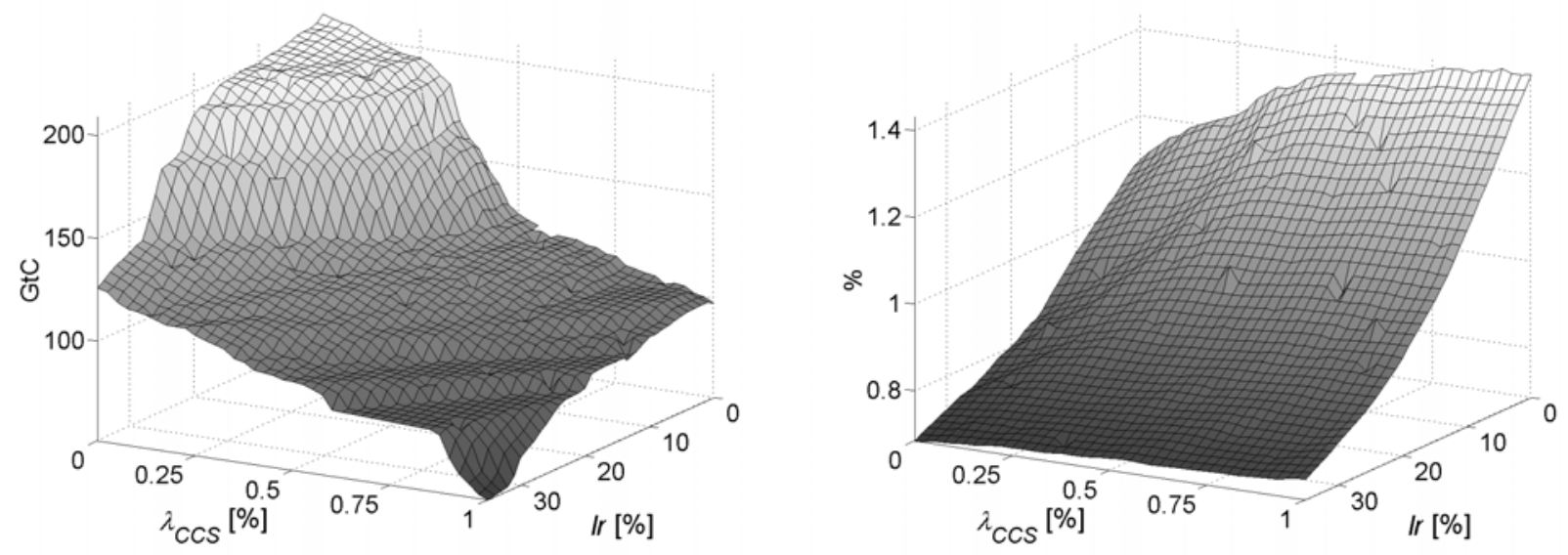

Fig. 2: Sensitivity of cumulative CCS (left panel) and cumulative discounted GDP losses (right panel) with respect to renewables learning rate $l r$ and leakage rateof sequestrtion sites $\lambda_{C C S} 2000-2050$.
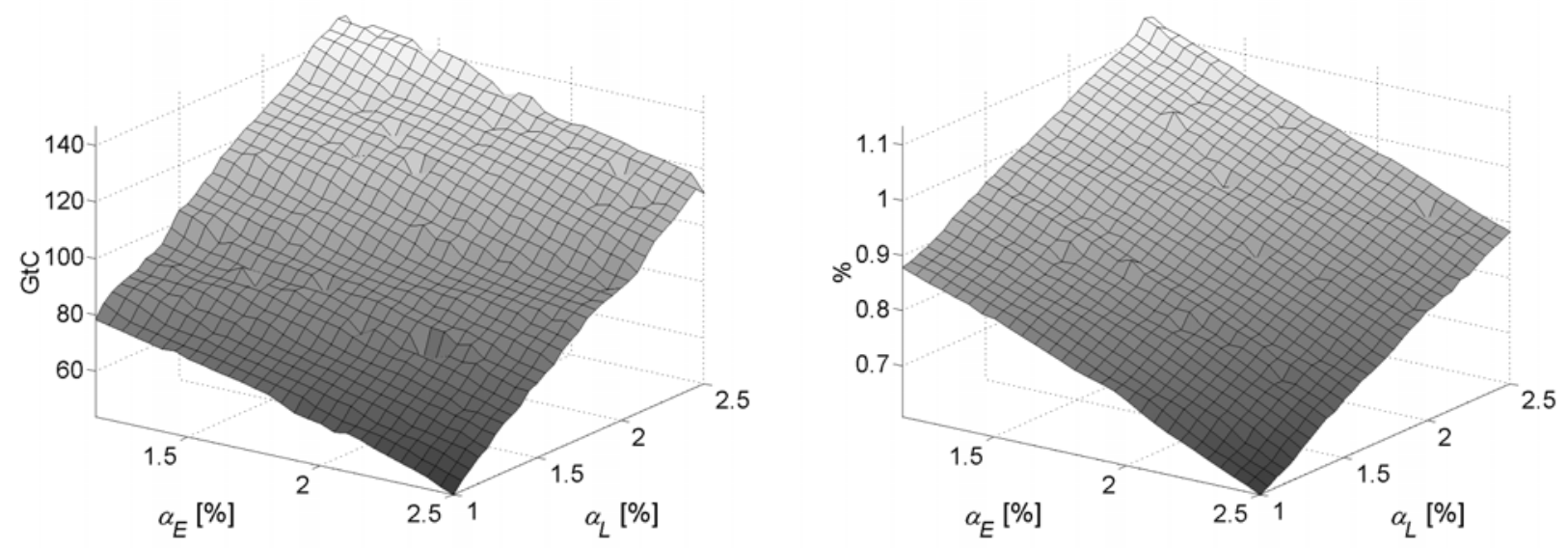

Fig. 3: Sensitivity of cumulative CCS (left panel) and discounted cumulative GDP losses (right panel) with respect to prameters improving the productivity of labour $\alpha_{L}$ and energy $\alpha_{E} 2000-2050$. 

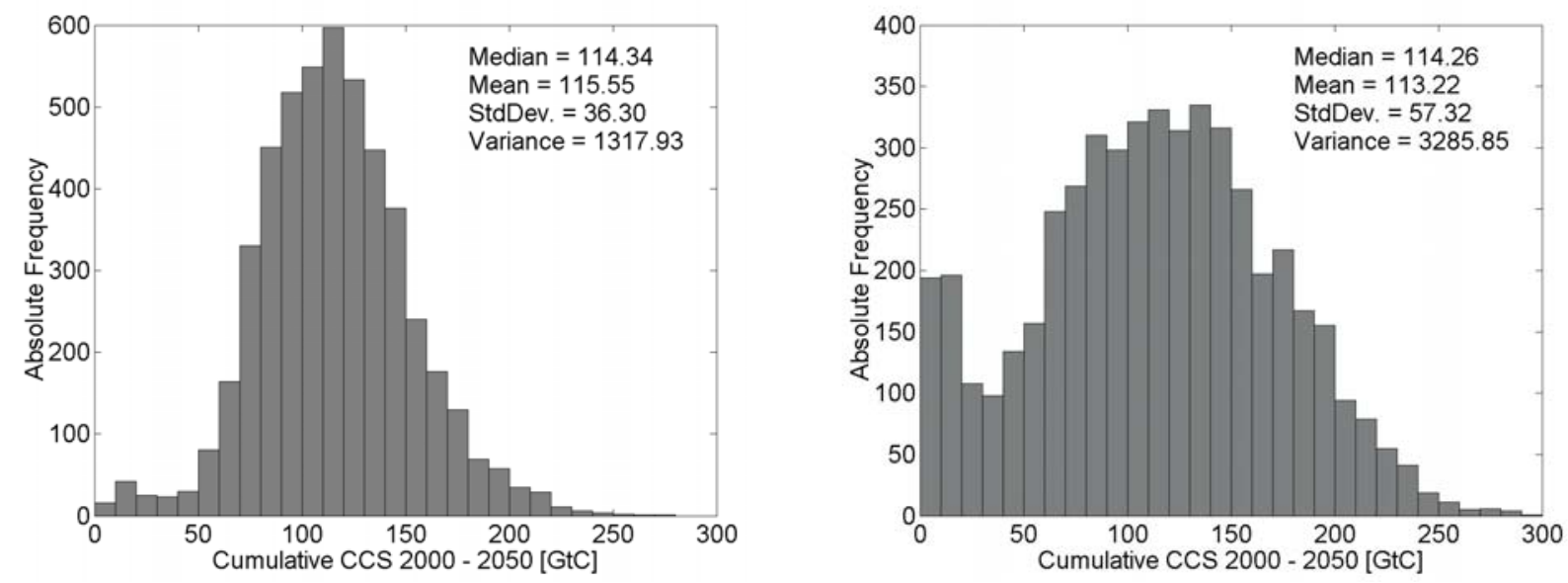

Fig. 4: Frequency distribution of cumulative CCS 2000 - 2050 for the Monte Carlo Analysis. The centers of the bins are the equivalent

\begin{tabular}{|l|l|l|l|l|l|l|l|l|l|}
\hline Parameter & $\alpha_{L}$ & $\alpha_{E}$ & $\sigma$ & $\kappa_{\text {res }, l}^{\max }$ & $K_{\text {fos }}^{0}$ & $K_{\text {res }}^{0}$ & $\chi$ & $c_{0}^{\text {ren }}$ & $l r$ \\
\hline Unit & non & non & non & non & tril.\$US & tril.\$US & GtC & $\$ \mathrm{US} / \mathrm{kW}$ & non \\
\hline Mean & 0.02 & 0.015 & 0.4 & 2 & 6 & 5 & 3500 & 700 & 0.15 \\
\hline Variance & $1.5 \mathrm{e}-5$ & $1.5 \mathrm{e}-5$ & $5 \mathrm{e}-3$ & $7.5 \mathrm{e}-2$ & 1 & 0.75 & $5 \mathrm{e}+5$ & $2.25 \mathrm{e}+4$ & $1 \mathrm{e}-3$ \\
\hline Parameter & $\phi_{\text {Hydrogen }}^{C C S}$ & $c_{\text {Hydrogen }}^{C C S}$ & $\phi_{\text {NewCoal }}^{C C S}$ & $c_{\text {NewCoal }}^{C C S}$ & $s_{C C S, S A O N}^{\max }$ & $s_{C C S, S A O F}^{\max }$ & $\lambda_{\text {CCS,SAON }}$ & $\lambda_{\text {CCS,SAOF }}$ & $\theta_{C C S, j}$ \\
\hline Unit & Non & $\$ \mathrm{US} / \mathrm{tC}$ & Non & $\$ \mathrm{US} / \mathrm{tC}$ & $\mathrm{GtC}$ & $\mathrm{GtC}$ & non & Non & $1000 \mathrm{~km}$ \\
\hline Mean & 0.06 & 98 & 0.2 & 156 & 2500 & 1000 & 0.005 & 0.005 & 0.15 \\
\hline Variance & $2 \mathrm{e}-4$ & 100 & $1.5 \mathrm{e}-3$ & 750 & $2.19 \mathrm{e}+5$ & $3.5 \mathrm{e}+4$ & $1 \mathrm{e}-6$ & $1 \mathrm{e}-6$ & $1.5 \mathrm{e}-3$ \\
\hline
\end{tabular}

Tab. 1: Parameters of distribution functions for high-dimensional Monte Carlo Analysis ( $\mathrm{SAON}=$ saline aquifer onshore, $\mathrm{SAOF}=$ saline aquifer off-shore). 